\title{
Priming 4.5-Month-Old Infants to Use Height Information by Enhancing Retrieval
}

\author{
Su-hua Wang \\ University of California, Santa Cruz
}

\begin{abstract}
How do infants select and use information that is relevant to the task at hand? Infants treat events that involve different spatial relations as distinct, and their selection and use of object information depends on the type of event they encounter. For example, 4.5-month-olds consider information about object height in occlusion events, but infants typically fail to do so in containment events until they reach the age of 7.5 months. However, after seeing a prime involving occlusion, 4.5 -month-olds became sensitive to height information in a containment event (Experiment 1). The enhancement lasted over a brief delay (Experiment 2) and persisted even longer when infants were shown an additional occlusion prime but not an object prime (Experiment 3 ). Together, these findings reveal remarkable flexibility in visual representations of young infants and show that their use of information can be facilitated not by strengthening object representations per se but by strengthening their tendency to retrieve available information in the representations.
\end{abstract}

Keywords: priming, infancy, object representation, physical reasoning, reminder

Experience plays an important role in perceptual and cognitive development. For example, naturally occurring motor experience influences spatial perception and search in infancy (e.g., Acredolo, Adams, \& Goodwyn, 1984; Bushnell \& Boudreau, 1993; Campos et al., 2000; Kellman \& Arterberry, 1998). Being able to sit up and to crawl increases the opportunities for infants to explore objects and thus enhances their ability to process and reason about objects (e.g., Soska, Adolph, \& Johnson, 2010). In addition to naturally occurring experience, deliberate manipulation of experiences at home or in the laboratory enhances infants' perception of and performance in dynamic events (e.g., Amso \& Johnson, 2006 Barrett, Davis, \& Needham, 2007; Hauf, Elsner, \& Aschersleben, 2004; Sommerville, Hildebrand, \& Crane, 2008; Sommerville, Woodward, \& Needham, 2005; Wang \& Kohne, 2007; Wilcox, Woods, \& Chapa, 2008).

In particular, a variety of enhancement effects have been observed on infants' processing of physical events (i.e., events that involve an object moving in relation to another object). First, enhancement effects can occur cross-modally. For example, action experience helps infants visually segregate and manually explore objects (e.g., Dueker, Modi, \& Needham, 2003; Needham, 2000; Needham, Barrett, \& Peterman, 2002). Second, enhancement effects can occur between similar events or features. For example,

This article was published Online First November 8, 2010.

This research was supported by a Research Grant from the Chiang Ching-kuo Foundation and by a Faculty Research Grant from UCSC. I thank Kris Onishi for helpful comments, Lili Beggs and Maggie Muir at the Sutter Santa Cruz Lactation Center for generous assistance with subject recruitment, and the families who kindly participated in the research.

Correspondence concerning this article should be addressed to Su-hua Wang, Department of Psychology, University of California, 1156 High Street, Santa Cruz, CA 95064. E-mail: suhua@ucsc.edu watching covering events in which various covers are lowered over an object guides infants to detect physical violations when they encounter another covering event (e.g., Wang \& Baillargeon, 2008a; Wang \& Kohne, 2007), and seeing an object feature, such as color, being highlighted helps infants individuate objects based on the feature (e.g., Wilcox \& Chapa, 2004; Wilcox, Woods, Chapa, \& McCurry, 2007). Finally, some effects of enhancement appear to generalize across contexts, demonstrating transfer of learning in infancy (e.g., Bahrick, 2002; Hayne, Barr, \& Herbert, 2003; Learmonth, Lamberth, \& Rovee-Collier, 2004). For example, watching occlusion events (i.e., an object moving in front of another object) in which infants have learned to use height information primes them to use the information in a different type of physical event: covering (Wang \& Baillargeon, 2005).

What is striking about the generalization from occlusion to covering events is that infants under a year of age often fail to apply what they learn in one event context to another. Instead, infants tend to treat events that involve different spatial relations (e.g., occlusion, containment, covering, and support) as distinct categories, even when they are perceptually similar (e.g., Baillargeon \& Wang, 2002; Casasola, 2005; Rigney \& Wang, 2009). For each event category, infants appear to identify certain object features (e.g., height, width, weight) as relevant variables for predicting the outcome of an event (e.g., whether an object should become fully or partly hidden when lowered behind another object, or whether an object should move or remain stationary when hit by another object). Infants readily use information about variables that have been identified as relevant when encountering new examples from the same category (e.g., Aguiar \& Baillargeon, 2002; Huettel \& Needham, 2000; Wang \& Baillargeon, 2006). For example, at the age of 3.5 months, infants appear to identify the variable height as relevant for predicting occlusion events, noticing that whether an object becomes fully or partially hidden behind a screen depends on the relative heights of the object and screen 
(Baillargeon \& DeVos, 1991). In addition, infants often apply variables specifically to the event context in which they initially learned about the variables and fail to generalize from one event category to another. As a result, they tend to use information about a particular variable in some but not other event categories (e.g., Hespos \& Baillargeon, 2001, 2006; Wang \& Baillargeon, 2006). For example, 4.5- to 6.5-month-olds detect a violation when a tall object becomes fully hidden behind a short container but not when the tall object becomes fully hidden inside the short container, suggesting that they use height information in occlusion but not containment events (Hespos \& Baillargeon, 2001). It is not until they are about 7.5 months of age that infants begin to use height information in containment events (Hespos \& Baillargeon, 2001, 2006).

Thus, whether infants detect a violation that involves a particular variable seems to depend on how they categorize the event and whether they have identified the variable as relevant and something they should consider (e.g., Baillargeon, Li, Ng, \& Yuan, 2009; Wang \& Baillargeon, 2008b). However, an exception has been observed in which infants' experience with one event category affects their processing of another category (e.g., Baillargeon et al., 2009; Wang \& Baillargeon, 2005). How might these crosscategory effects occur? For example, how might infants be primed, through watching occlusion events, to consider height information in covering events (Wang \& Baillargeon, 2005)? A three-system model was put forward to explain these findings. According to this model, three computational systems are involved when infants represent a physical event that they observe (Wang \& Baillargeon, 2008b). An object-representation system encodes and stores information about each object involved, an object-tracking system assigns to each object an index that serves as a pointer and allows infants to trace the object as it moves (e.g., Leslie, Xu, Tremoulet, \& Scholl, 1998; Pylyshyn, 2001; Scholl \& Leslie, 1999), and a physical reasoning system enables reasoning about object interactions by categorizing events and specifying what object features that infants have come to know as relevant for predicting outcomes and as something that should be bound to the object index.

Consider Wang and Baillargeon's (2005) report, in which 8-month-old infants watched a cover being moved in front of an object (occlusion event) and then lowered over the object (covering event). Although infants typically fail to detect a violation when a short cover fully hides a tall object until they are about 12 months of age, the 8-month-olds who watched the occlusion event prior to the covering event succeeded in detecting the violation. How does the three-system model explain this finding? According to this model, upon noticing the cover and object, an index would be assigned to each stimulus and featural information (including height) would be encoded and stored in the object-representation system. As soon as the cover was moved in front of the object, the physical reasoning system built a representation about the event. It first bound basic information about each stimulus (e.g., was the box open or closed at the top?) to each index. Once the object was moved to the front of the box, the physical reasoning system used this spatial information to determine the event category, in this case, occlusion. Next, the physical reasoning system tapped infants' existing knowledge about the event category. At 8 months of age, infants already learn that height is a relevant variable for predicting the outcomes of occlusion events (infants become sen- sitive to this variable in occlusion events at about 3.5 months; see Baillargeon \& DeVos, 1991). Therefore, height information about each stimulus was retrieved from the object-representation system and bound to each object index. Immediately after the occlusion event, the infants watched the covering event that involved the same stimuli. Because the two events involved the same objects and occurred consecutively without any delay, the same object indexes were used for processing both events. As the indexes were traced from the first to the second event, height information bound to the indexes was carried forward from the representation of the first event to that of the second event. Thus, even though infants typically do not include height information in their representations of covering events until 12 months of age (e.g., Wang \& Baillargeon, 2006), the 8-month-olds who watched occlusion events were primed to do so, making it possible for them to detect the violation (Wang \& Baillargeon, 2005).

The three-system account makes a few predictions regarding the priming phenomenon. First, it predicts that priming should occur between any pair of event categories and with younger infants, as long as infants have identified the variable as relevant for the first event and as long as the second event involves the same objects and occurs immediately after the first so that the same indexes can be used to track objects across events. Second, it predicts that priming should dissipate when object indexes have faded or when featural information is no longer bound to the indexes. Thus, when a delay long enough for indexes or bound information to fade is inserted between the two events, infants should no longer benefit from priming. Third, in the case where priming has dissipated due to a long delay, the account predicts that exposure to another occlusion event during the delay should prompt infants to bind variable information to the indexes again and thereby enable them to use the information when they watch the second event after the delay. In the present research, we tested these predictions in an attempt to deepen our understanding about the representational process in infancy and about the effects of experience on the process.

Experiment 1 examined whether priming could occur in another pair of event categories, from occlusion to containment, and with younger infants who had just learned about the relevance of height for occlusion events. Experiment 2 addressed the issue of robustness by exploring how long the indexes and bound information lasted. Finally, Experiment 3 tested the third prediction and examined whether exposure to another occlusion event would enable infants to use height information even after a long delay.

\section{Experiment 1}

After seeing an occlusion prime, 8-month-olds were induced to detect a violation of height in a covering event (Wang \& Baillargeon, 2005). The three-system model predicts that similar priming results should be obtained from occlusion to containment. As demonstrated by Hespos and Baillargeon (2001), infants begin to identify height as a relevant variable for containment events at about 7.5 months, but they appear to recognize that height is relevant for predicting the outcomes of occlusion events at 4.5 months of age. Experiment 1 attempted to (a) replicate this developmental change and (b) test whether watching occlusion events primes 4.5 -month-olds to consider height information in containment events. 
To replicate the developmental pattern shown in Hespos and Baillargeon (2001), 4.5- and 8-month-old infants (replication groups) received two brief trials in which they watched target events (see Figure 1, Target Event). In each target event, an object was lowered inside a container and became fully hidden. The container was slightly taller than the object in one trial (plausible event; tall-container trial) and much shorter in the other trial (anomalous event; short-container trial).

To test whether watching occlusion events would prime infants to detect the violation involving height in containment events, two groups of 4.5-month-olds (priming groups) were tested as the replication groups described above, except that immediately before the target events the infants watched priming events (see Figure 1, Priming Event). Half of the infants watched occlusion priming events, whereas the other half watched nonocclusion events. In the occlusion priming events, the object was first moved in front of the container and then returned to its starting position. In the nonocclusion priming events, the object was moved to make contact with the side of the container but never occluded it, and then returned to its starting position. Both of the above sequences were repeated six times in the priming events.

According to the previous findings (Hespos \& Baillargeon, 2001), a developmental change should be observed in the replication groups. That is, 8-month-olds should detect the violation and look reliably longer in the short- than in the tall-container trial, whereas 4.5-month-olds should fail to notice the violation and look about equally in the two trials. The priming groups should also respond differently to the target events, depending on the prime they received. According to the three-system model, after watching the occlusion prime, height information should be bound to each object index. As the 4.5-month-olds watched the priming and target events consecutively, without any delay, the same indexes would be used to track the objects across events. As a result, height information bound to the indexes should be transferred from their representations of occlusion events to those of containment events, allowing them to detect the violation in the short-container trial. Thus, the infants in the occlusion prime condition should look reliably longer in the short- than in the tall-container trial. In contrast, height is not a relevant variable for predicting the outcomes of events that involved an object making contact with another object (e.g., Baillargeon et al., 2009). Therefore, there was no reason for the infants who received the nonocclusion prime to bind height information to object indexes, even though the object was shown in the priming events for the same duration as in the occlusion prime condition. Because height information was not bound to object indexes in the first place, it could not be carried forward to infants' representations of the target events. Thus, the infants in the nonocclusion prime condition should fail to detect the violation as they normally would and should therefore look equally in the two trials.

\section{Method}

Participants. Participants in this and following experiments were healthy term infants, who were primarily Caucasian and from middle-class backgrounds. The replication groups included 14 8 -month-old infants ( 7 female, 7 male; range $=7$ months 16 days to 8 months 20 days; $M=8$ months 1 day) and 144.5 -month-old infants ( 6 female, 8 male; range $=4$ months 1 day to 5 months 17 days; $M=4$ months 20 days). The priming groups included 32 4.5-month-old infants ( 14 female, 18 male; range: 4 months 0 days to 5 months 12 days); half were randomly assigned to the occlusion prime condition ( $M=4$ months 21 days) and half to the nonocclusion prime condition ( $M=4$ months 27 days). An additional 18 infants (including two 8-month-olds and 16 4.5-montholds) were tested, but their data were eliminated for looking for the
Tall-Container Trial

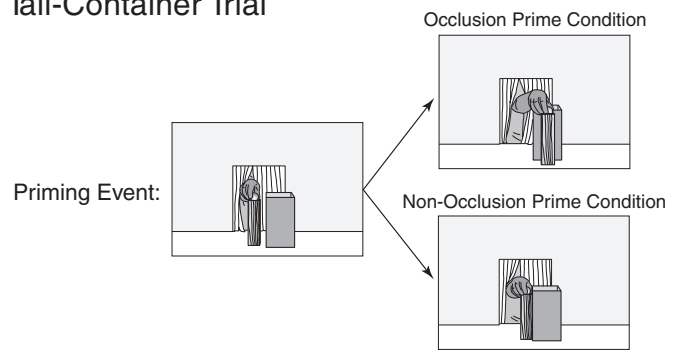

Short-Container Trial

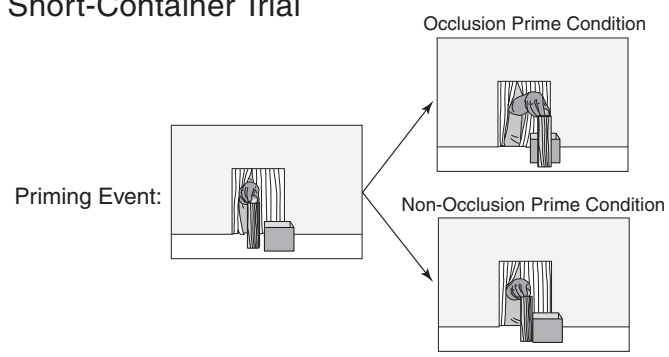

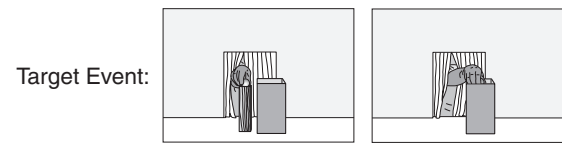

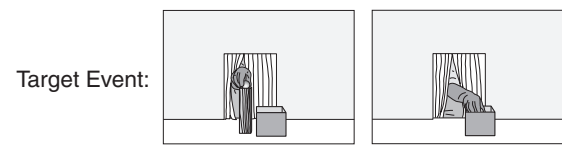

Figure 1. Schematic drawings of the priming and target events in Experiment 1. 
maximum time allowed on both trials $(n=8),{ }^{1,2}$ inattentiveness or fussiness $(n=9)$, or parental interference $(n=1)$. The infants' names were obtained from hospitals or birth announcements published in newspapers. Parents received a small gift or travel reimbursement but were not otherwise compensated for participation.

Apparatus. A wooden display box $(104 \mathrm{~cm}$ wide $\times 106.3 \mathrm{~cm}$ high $\times 56.3 \mathrm{~cm}$ deep) was mounted $76 \mathrm{~cm}$ above the room floor. The infants faced an opening $(97 \mathrm{~cm} \times 45 \mathrm{~cm})$; between trials, a curtain was lowered in front of it. An experimenter introduced the right hand in a long black glove into the apparatus through a window $(27.5 \mathrm{~cm} \times 35 \mathrm{~cm})$ centered in the back wall; the window extended from the bottom of the wall and was concealed by white ribbons.

The stimuli included an object and two containers. The object was made of wood $(5 \mathrm{~cm} \times 18 \mathrm{~cm} \times 4.7 \mathrm{~cm})$ with a knob $(2 \mathrm{~cm}$ high) affixed on its top. The containers were made of cardboard, $11 \mathrm{~cm}$ wide and $10 \mathrm{~cm}$ deep. To ensure that the infants noticed that they were facing a container, the back wall of each container was slightly taller than the front wall, and the side walls angled slightly to join them; each wall was $0.5 \mathrm{~cm}$ thick. The tall container was blue, $19 \mathrm{~cm}$ high in front, and $21 \mathrm{~cm}$ high in back; the short container was green, $9 \mathrm{~cm}$ in front, and $10 \mathrm{~cm}$ in back. The interior of each container was white.

The infants were tested in a brightly lit room. Four $60-\mathrm{W}$ lamps attached to the apparatus provided additional light. Two fabriccovered frames (each $71 \mathrm{~cm} \times 182.3 \mathrm{~cm}$ ) were hinged at an angle on either side of the apparatus, isolating infants' view from the rest of the room.

Events. The replication groups watched target events only, whereas the priming groups watched priming and target events consecutively. The priming events in the occlusion and nonocclusion prime conditions differed slightly, whereas the target events were identical across conditions. Each infant received two trials: one trial was conducted with the short container and the other with the tall container. In the following section, the numbers in parentheses indicate the time taken to perform each action. A metronome beat softly once per second to help the experimenter follow the scripts for the events.

Priming events. In the occlusion prime condition, at the start of the trial, the object stood centered $2 \mathrm{~cm}$ to the left of the container with the experimenter grasping the knob (see Figure 1). When the infant had looked at this display for two cumulative seconds, the experimenter slid the object $10 \mathrm{~cm}$ toward the infant (1 s). Next, the object was slid $10 \mathrm{~cm}$ to the right so that it stood centered in front of the container ( $1 \mathrm{~s})$; after a pause (1 s), it was slid $10 \mathrm{~cm}$ to the left $(1 \mathrm{~s})$, followed by another pause (1 s). The 4-s sequence was repeated six times; thus, the priming event, which consisted of a sequence of six occlusions, lasted $24 \mathrm{~s}$.

Different from the occlusion prime in Wang and Baillargeon (2005), in which an object was either partly or fully hidden behind a cover, the occlusion prime in Experiment 1 involved an object being moved in front of a container. This new design ensured that the object remained fully visible in both short- and tall-container trials, which increased the similarity between the two trials and eliminated a potential confounding variable that infants might respond differently to the target events depending on whether the object was fully or partly hidden in the priming events. ${ }^{3}$ In other words, the design alleviated a potential concern that the priming effects, if obtained, might reflect infants' sensitivity to differing amounts of changes to the physical appearance of the object (i.e., from fully visible to fully hidden versus from fully visible to partly visible), rather than infants' increased ability to use height information.

In the nonocclusion prime condition, after the infant had accumulated $2 \mathrm{~s}$ of looking at the starting display, the experimenter moved the object $8 \mathrm{~cm}$ to the left away from the container so that the object stood $10 \mathrm{~cm}$ away from the left side of the container (1 s). Next, a sliding sequence was conducted: The object was slid 10 $\mathrm{cm}$ to the right to contact the side wall of the container $(1 \mathrm{~s})$; after a pause $(1 \mathrm{~s})$, the object was slid $10 \mathrm{~cm}$ to the left $(1 \mathrm{~s})$, followed by another pause $(1 \mathrm{~s})$. The above 4 -s sliding sequence was repeated six times; thus, this version of the priming event, consisting of a sequence of six nonocclusion events, also lasted $24 \mathrm{~s}$. Note that the sliding movement was identical across conditions, and the two conditions differed only in the location of the movement. In the nonocclusion prime condition, the movement occurred next to the container, eliminating the occlusion component.

Target events. For the priming groups, the experimenter first slid the object back to its starting position, centered $2 \mathrm{~cm}$ to the left of the container $(1 \mathrm{~s})$. For the replication groups, this movement was replaced by a 1-s pause, given that infants did not watch any priming event and the object already stood at this location at the beginning of the target events. Next, for both groups, the experimenter lifted the object (1 s), moved it to the right until it was directly above the container ( $1 \mathrm{~s}$ ) and lowered it inside the container ( $2 \mathrm{~s}$ ) until only the top centimeter of the knob was visible above the front wall of the container (following Hespos \& Baillargeon, 2001). ${ }^{4}$ After a pause (1 $\mathrm{s})$, the object was lifted ( $2 \mathrm{~s})$, moved to the left (1 s), and lowered to its starting position $(1 \mathrm{~s})$, followed by another pause (1 s). The 10-s sequence (starting from lifting the object) was repeated until the trial ended (see the following).

\footnotetext{
${ }^{1}$ Even if these max-out infants were included, the analysis of Experiment 1 still yielded similar results (see Results and Discussion).

${ }^{2}$ It is a common practice in violation-of-expectation research to eliminate from analysis infants who look at both expected and unexpected events for the maximum amount of time allowed (e.g., Hespos \& Baillargeon, 2001; Luo \& Baillargeon, 2007; Wang \& Baillargeon, 2005; Wilcox et al., 2007), because they fail to provide interpretable data. A number of reasons could underlie such responses. Infants may do so because they fail to detect the violation and instead are captured by other aspects of the events, or these "long lookers" may respond with differing looking durations if extra time is given. However, when infants are separated into two groups and only watch one type of events (expected or unexpected), max-out responses become interpretable with the between-subjects design: If such responses are derived not from detecting a violation, it should be observed equally often in infants who saw expected and those who saw unexpected events. This hypothesis can be tested by comparing the looking pattern across the two groups of infants.

${ }^{3}$ Wang and Baillargeon (2005, Experiment 2) eliminated the potential confound of differing outcomes in priming events by using an object that was shorter than either cover, in addition to the original, tall object. The experimenter first slid the cover in front of the very short object, creating a fully hidden outcome whether the short or the tall cover was used; next, the cover was lowered over the tall object. Converging evidence was obtained: Exposure to occlusion events induced the infants to use height information in covering events (Experiment 2).

${ }^{4}$ Making the knob visible throughout the experiment helps lessen the task demand for these young infants. It provided a reference point for the presence of the object when its main portion became fully hidden inside the container.
} 
Procedure. Each infant sat on the parent's lap about $45 \mathrm{~cm}$ in front of the lowered curtain. Parents were instructed to remain quiet and to keep their eyes closed during the trials. Each infant received two test trials, a tall- and a short-container trial conducted with either the tall or short container. The order of the trials was counterbalanced across infants.

Two observers, blind to the research hypothesis and unable to see the events, watched the infants through peepholes in the fabric-covered frames on either side of the apparatus. Each observer pressed a button linked to a computer when the infants looked at the events. Observers also noted each infant's state (e.g., whether the infant appeared drowsy, quiet and attentive, active, or fussy). The looking times recorded by the primary (typically more experienced) observer were used to determine the end of the trials. Each trial ended when the infants (a) looked away from the target event for 1.5 consecutive seconds after having looked at it for at least 10 cumulative seconds or (b) looked at it for 80 cumulative seconds. The 10-s minimum value was chosen to ensure that infants had the opportunity to watch at least one cycle of the event.

Each trial was divided into 100-ms intervals, and the computer determined in each interval whether the two observers agreed on infants' gaze direction (i.e., looking at the event or not). Interobserver agreement during the target events was calculated by dividing the number of intervals in which observers agreed by the total number of intervals. It ranged from $86 \%$ to $100 \%$ and averaged $96 \%$ per infant per trial.

\section{Results and Discussion}

In this and the following experiments, preliminary analyses did not yield any significant interaction involving trial type and sex or trial type and trial order (all $F \mathrm{~s}<2.03, p \mathrm{~s}>.10$ ). Therefore, the data were collapsed across sex and trial order in subsequent analyses.

Replication groups. The infants' looking times at the target events (see Figure 2) were compared by a $2 \times 2$ analysis of variance (ANOVA) with age (8- or 4.5-month-olds) as a betweensubjects factor and trial type (tall- or short-container trial) as a within-subject factor. The analysis yielded a significant Age $\times$ Trial Type interaction, $F(1,26)=4.99, p<.05$. Planned comparisons indicated that the 8-month-olds looked reliably longer in the short- $(M=38.91 \mathrm{~s}, S E=5.37 \mathrm{~s})$ than in the tall-container trial $(M=25.98 \mathrm{~s}, S E=2.98 \mathrm{~s}), F(1,26)=5.12, p<.05$, Cohen's $d=$ 0.79 , whereas the 4.5 -month-olds looked about equally in the two trials (short-container $M=28.06 \mathrm{~s}, S E=4.29 \mathrm{~s}$; tall-container $M=33.13 \mathrm{~s}, S E=4.38 \mathrm{~s}), F(1,26)=0.77, d=0.21$. Nonparametric Wilcoxon signed-ranks tests confirmed these results (8month-olds $T=11, p<.05 ; 4.5$-month-olds $T=43, p>.10$ ).

Another analysis was conducted with the addition of the three infants who looked at both events for $80 \mathrm{~s}$ (one 8-month-old and two 4.5-month-olds), and it yielded similar results. The Age $\times$ Trial Type interaction was again significant, $F(1,29)=5.09, p<$ .05 . Planned comparisons indicated that the 8-month-olds looked reliably longer in the short- $(M=41.65 \mathrm{~s}, S E=5.37 \mathrm{~s})$ than in the tall-container trial $(M=29.58 \mathrm{~s}, S E=2.98 \mathrm{~s}), F(1,29)=5.27$, $p<.05, d=0.74$, whereas the 4-month-olds did not (shortcontainer $M=34.56 \mathrm{~s}, S E=5.32 \mathrm{~s}$; tall-container $M=38.99 \mathrm{~s}$, $S E=5.19 \mathrm{~s}), F(1,29)=0.76, d=0.19$.

The results indicated a developmental change in infants' responses to containment events. Whereas 4.5-month-olds were not
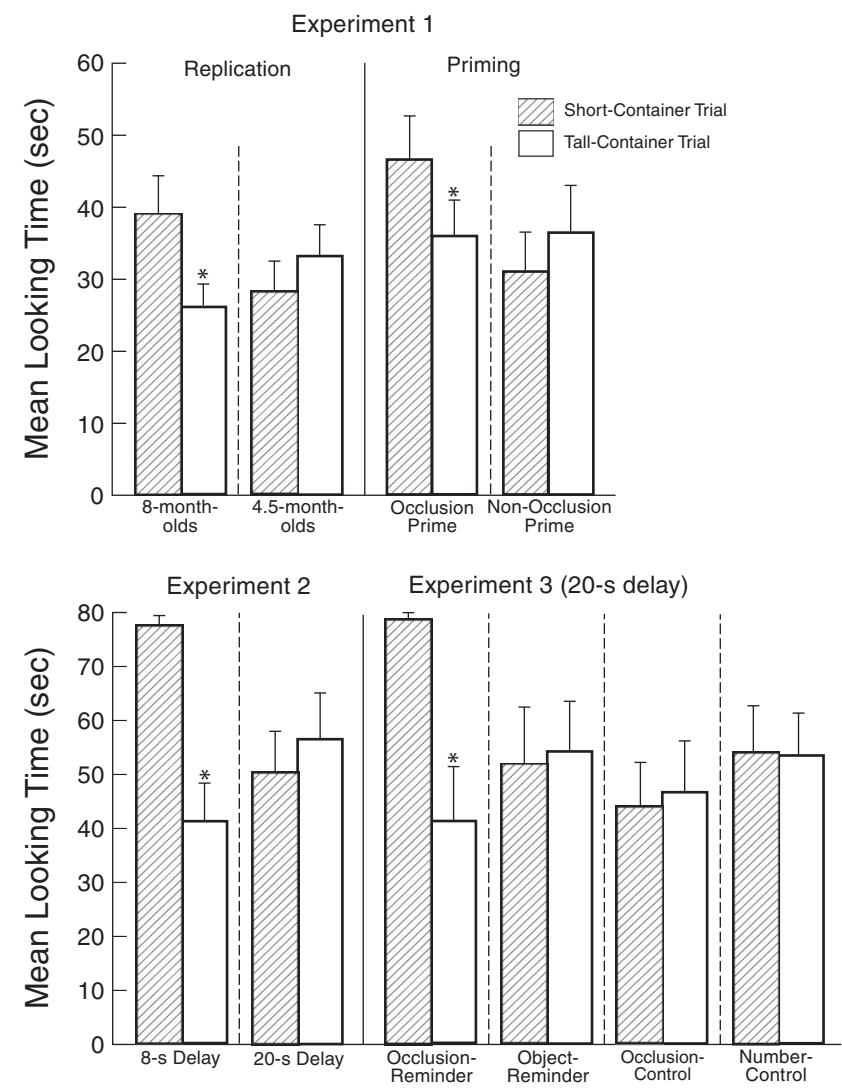

Figure 2. Infants' mean looking times at the target, containment event in Experiments 1 to 3 . Striped bars represent the looking times in the shortcontainer trial, and white bars the tall-container trial. Error bars represent standard errors. Asterisks represent significant effects of trial.

sensitive to the violation in which a tall object became fully hidden inside a short box, 8-month-olds responded with heightened interest, as indicated by their prolonged looking time. The results echoed previous findings (Hespos \& Baillargeon, 2001, 2006), suggesting that infants at 8 but not 4.5 months of age readily use height information when watching containment events. Past research has highlighted the benefit of motor development in spatial and object perception (e.g., Bushnell \& Boudreau, 1993; Campos et al., 2000; Soska et al., 2010). Consistent with this view, it is likely that from 4.5 to 8 months of age, infants' increased ability to sit up and crawl provides them with increased opportunities to watch and act on containment events, resulting in a better use of object information in such event contexts.

Priming groups. The infants' looking times at the target events (see Figure 2 ) were compared by a $2 \times 2$ ANOVA with condition (occlusion or nonocclusion prime) as a between-subjects factor and trial type (tall- or short-container trial) as a withinsubject factor. The analysis yielded a significant Condition $\times$ Trial Type interaction, $F(1,30)=5.81, p<.025$. Planned comparisons indicated that the infants in the occlusion prime condition looked reliably longer in the short- $(M=46.32 \mathrm{~s}, S E=6.09 \mathrm{~s})$ than in the tall-container trial $(M=35.88 \mathrm{~s}, S E=5.07 \mathrm{~s}), F(1,30)=5.04$ $p<.05$, Cohen's $d=0.56$, whereas those in the nonocclusion prime condition looked about equally in the two trials (short- 
container $M=30.96 \mathrm{~s}, S E=5.43 \mathrm{~s}$; tall-container $M=36.37 \mathrm{~s}$, $S E=6.54 \mathrm{~s}), F(1,30)=1.35, p>.10, d=0.31$. Nonparametric Wilcoxon signed-ranks tests confirmed these results (occlusion prime $T=29, p<.05$; nonocclusion prime $T=54, p>.10){ }^{5}$

The 4.5-month-olds in the occlusion prime condition detected the violation in the containment event just as the 8-month-olds did, whereas the 4.5-month-olds in the nonocclusion prime condition still failed to detect the violation. Because the same stimuli were used in the occlusion and nonocclusion prime conditions, it is unlikely that prolonged looking was driven by infants' intrinsic bias toward some perceptual aspects of the short container. If this was the case, the infants in the nonocclusion prime condition should have looked longer in the short- than in the tall-container trial as well. But they did not. Thus, these results supported two conclusions. First, after watching occlusion events, the 4.5-montholds seemed to be primed to use height information in containment events, 3 months earlier than they would spontaneously do so. Second, priming was not derived from mere exposure to the object; exposure of the same duration in a nonocclusion event did not yield the same results. These findings thus supported the first prediction of the three-system model described in the introduction and helped establish the generality of priming by demonstrating the phenomenon between a new pair of event categories and with younger infants.

Taking the findings in a developmental perspective, Experiment 1 demonstrates that with the right kind of exposure, a single trial is sufficient to induce 4.5-month-olds to use height information in containment events - an ability that would otherwise not emerge until the age of about 8 months. Do infants encounter these kinds of priming events in their daily experience as they learn about containment events between 4.5 and 8 months of age? When infants act upon objects and watch others do so, it is likely that they observe different types of physical events sequentially, one type after another. Although empirical evidence on infants' daily observation of physical events is still needed, occlusion and containment events are fairly prevalent in their observational and action experience. However, even if the two types of events occur temporally close to each other, it seems less likely for infants to observe a containment event immediately preceded by an occlusion event (i.e., without any delay). Instead, a delay is likely to separate infants' observation of the two events in their everyday life. Therefore, to link the present priming findings to infants' everyday experience, it is important to examine whether the priming effects last after a delay.

\section{Experiment 2}

Although recent research demonstrates that infants' use of object information can be facilitated by various types of experiences, much less is known about the robustness of the enhancement. Given that 4.5-month-olds can be primed to use height information in containment events, it is possible to ask how long the enhancement would last. In adults, object indexes persist over an 8-s delay at least (Noles, Scholl, \& Mitroff, 2005). If the indexing system is similar in adults and infants, we would expect the priming effect to last over such a delay. Experiment 2 examined whether the effect of priming lasted several seconds in infants. Infants at 4.5 months watched the occlusion prime as in Experiment 1, except that a delay of either 8 or $20 \mathrm{~s}$ was inserted between the priming and target events.

\section{Method}

Participants were 28 healthy term infants ( 13 female; range $=3$ months 23 days to 5 months 18 days); they were assigned to the 8-s or the 20-s delay condition $(M=4$ months 24 days in both conditions). The procedure was similar to the occlusion prime condition of Experiment 1, with an additional step. At the end of the priming events, the parent who sat on a swivel chair turned the infant clockwise 180 degrees away from the apparatus so that they both faced a white wall. Parents were instructed to interact with infants only when it was necessary to keep infants in position. After the delay, the parent turned the chair counterclockwise 180 degrees to position the infant at his or her initial seating orientation, facing the apparatus.

Pilot data indicated that many infants became restless or fussy during the second trial, probably because of the lengthened duration of the procedure, resulting in several early terminations of the second trial. Thus, a between-subjects design was used in Experiment 2. Indeed, when required to complete only one test trial, none of the participating infants had to be eliminated from the analysis. Note that the infants who looked at the event for the maximum amount of time allowed were not excluded from the analysis in this and the following experience because of the between-subjects design (see Footnote 2 for the underlying rationale). Recall that the results in Experiment 1 showed no difference whether max-out infants were included or not. Thus, it is unlikely that including max-out infants in Experiment 2 will produce any artificial results.

Prior to the experiment, the parent practiced turning the swivel chair with the infant sitting on his or her lap and was told that during the experiment a supervisor would say "go" to signal him or her to turn. Specifically, the parent should turn the infant away from the apparatus at the first signal and back toward the apparatus at the second signal. During the trial, the supervisor said "go" on the 24th second (the last second) of the priming event to prompt the parent to turn the infant away facing the white wall. On the 8th or 20th second of the delay, the supervisor said "go" to signal the parent to turn the infant back facing the apparatus. ${ }^{6}$ Throughout the trial, the observers continuously monitored the infant's gaze direction and judged whether the infant was looking at the event area. None of the infants looked at the event area during the delay.

\footnotetext{
${ }^{5}$ Another set of analysis was conducted with the five max-out infants (three from the occlusion prime and two from the nonocclusion prime condition) and again yielded a significant Condition $\times$ Trial Type interaction, $F(1,35)=5.59, p<.025$. Planned comparisons showed that the occlusion prime group looked reliably longer in the short- $(M=51.64 \mathrm{~s}$, $S E=6.24 \mathrm{~s})$ than in the tall-container trial $(M=42.84 \mathrm{~s}, S E=5.59 \mathrm{~s})$, $F(1,35)=4.81, p<.05, d=0.51$, whereas the nonocclusion prime group did not (short-container $M=36.41 \mathrm{~s}, S E=6.04 \mathrm{~s}$; tall-container $M=$ $41.22 \mathrm{~s}, S E=6.86 \mathrm{~s}), F(1,35)=1.36, p>.10, d=0.28$.

${ }^{6}$ The delay ranged from $8.0 \mathrm{~s}$ to $10.7 \mathrm{~s}$ in the 8 -s delay condition $(M=$ $8.5 \mathrm{~s})$ and from $20.0 \mathrm{~s}$ to $30.1 \mathrm{~s}$ in the 20 -s delay condition $(M=22.0 \mathrm{~s})$. The variation of the delay was due to different speeds with which the parents made the turns. The supervisor signaled the experimenter to start conducting the target event by saying "go" after the infant had looked at the event area (indicated by the computer when the primary observer pressed the button). Thus, whether the delay was greater than $8 \mathrm{~s}$ or $20 \mathrm{~s}$ or not, all infants saw the beginning of the target event.
} 
Interobserver agreement during the target events ranged from $85 \%$ to $100 \%$ and averaged $96 \%$ per infant.

\section{Results and Discussion}

The infants' looking times at the target events (see Figure 2) were compared by a $2 \times 2$ ANOVA with condition (8-s or 20-s delay) and trial type (tall- or short-container trial) as betweensubjects factors. The analysis yielded a significant main effect of trial type, $F(1,24)=4.75, p<.05$, and a significant Condition $\times$ Trial Type interaction, $F(1,24)=9.64, p<.005$. Planned comparisons indicated that in the 8-s delay condition, the infants who received the short-container trial looked reliably longer $(M=$ $77.30 \mathrm{~s}, S E=1.95 \mathrm{~s})$ than did those who received the tallcontainer trial $(M=41.09 \mathrm{~s}, S E=6.95 \mathrm{~s}), F(1,24)=13.95, p<$ .001 , Cohen's $d=2.68$. In the 20-s delay condition, however, the infants looked about equally (short-container $M=50.11 \mathrm{~s}, S E=$ $7.77 \mathrm{~s}$; tall-container $M=56.46 \mathrm{~s}, S E=8.69 \mathrm{~s}), F(1,24)=0.43$, $d=0.29 .^{7}$ Nonparametric Wilcoxon rank-sum tests confirmed these results (8-s delay $W=32.5, p<.025 ; 20$-s delay $W=48$, $p>.10)$. Thus, the infants in the 8 -s delay condition used height information to detect the violation in the containment event, whereas those in the 20-s delay condition did not. This finding suggested that priming in 4.5-month-olds lasted at least $8 \mathrm{~s}$ and dissipated by $20 \mathrm{~s}$.

Experiment 1 showed that watching occlusion events primed 4.5-month-olds to consider height information in containment events, whereas Experiment 2 provided evidence that infants still benefited from priming when a delay of 8 (but not 20) s was inserted between priming and target events. Based on the threesystem model, watching occlusion events prompts infants to retrieve relevant featural information from the object-representation system and bind the information to object indexes. When infants track objects from the first to the second event via the same object indexes, featural information bound to these indexes is transferred from the representation of the priming event to that of the target event, enabling infants to detect the violation (e.g., Wang \& Baillargeon, 2008b). Accordingly, this model provides three possible explanations for why priming dissipates after the 20-s delay.

The first explanation pertains to how long the object index lasts. Although the index may persist for a few seconds after the object is out of infants' view, eventually it fades away. Based on the model, height information is carried over from the representation of the first event to that of the second event, because the same index is used to trace the object across events. When the index no longer exists, infants have to assign a new index when they see the object again after the delay and during the containment event; this time, there is no occlusion component in the event to activate the binding of height information to the index. As a result, 4.5-montholds fail to detect the violation.

The second explanation pertains to how long the represented information lasts. Perhaps, 4.5-month-old infants are unable to maintain a strong representation of the object when it has become out of view for many seconds. In this case, even if the index is still available after the delay for infants to trace the object and is carried forward from the first to the second event, bound information cannot be carried forward when it is no longer available in the representation of the first event. Both explanations discussed so far predict that if the object remains in view during the 20-s delay, the index or bound information should be maintained. Thus, continued exposure to the stimuli during the delay should lessen the dissipation of priming effects and allow infants to use height information in the containment event. Experiment 3 tested this prediction (object-reminder condition).

A third possible explanation pertains to how long the binding lasts. It could be that after a long delay, both the index and information are available somewhere in the representation, but they are no longer bound together. Consequently, height information cannot be carried over. As discussed in the introduction, the model predicts that if infants watch another priming event during the delay, variable information should be bound to the index again, allowing infants to use the information even after a long delay. Experiment 3 tested this prediction by showing infants additional occlusion events that involved a different set of stimuli during the 20-s delay (occlusion-reminder condition). Positive results would suggest that priming can be reiterated by the exposure to an additional prime and that watching priming events involving one set of stimuli could enable infants to use relevant information about another set of stimuli.

\section{Experiment 3}

The 4.5-month-olds in Experiment 2 failed to detect the violation involving height information in containment events when time had elapsed $20 \mathrm{~s}$ since they watched occlusion priming events. Experiment 3 asked whether (a) continued exposure to the stimuli or (b) an occlusion reminder involving different stimuli could extend the effect of priming beyond the 20-s delay. Infants first watched occlusion events as in Experiments 1 and 2. Next, a 20-s delay was introduced, during which the infants received one of two reminders (see Figure 3). After the delay, the infants watched target events as in Experiments 1 and 2.

In the object-reminder condition, infants watched the object being slid back and forth at the left side of the container during the delay. If priming dissipated because the object index had faded or because height information had become unavailable in the representation after the object became out of view for too long, then continued exposure to the object (with motion to engage infants' attention) should prevent the index or information from fading and should allow infants to detect the violation after a long delay. In contrast, if priming dissipated because information was no longer bound to the object index, then continued exposure to the object without seeing any occlusion should not help infants detect the violation.

In the occlusion-reminder condition, infants watched occlusion events involving a second object (Object 2) and a screen during the

\footnotetext{
${ }^{7}$ The average looking times of Experiment 2 were higher than those of Experiment 1, as a result of different designs. Recall that infants in Experiment 1 received two trials; thus, each trial type was administered as the first trial for half of the infants and as the second trial for half of the infants. Because infants' looking time generally decreases over the course of multiple trials, the looking times of Experiment 2 collected in the first trial tended to be higher than the looking times of Experiment 1 averaged from two trials. The elevated looking times in Experiment 2 were perhaps also due to infants' recovered interest in the stimuli after having looked at the white wall during the delay. However, this factor should not affect the short- and tall-container trials differently and hence could not account for infants' different responses across the two trials.
} 
Occlusion-Reminder Condition

Tall-Container Trial

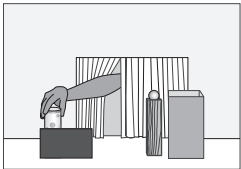

Short-Container Trial

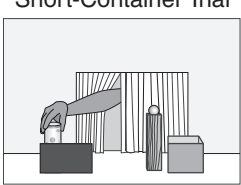

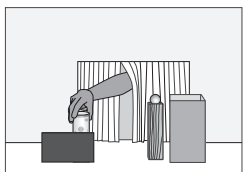

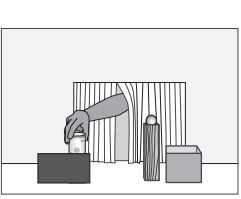

Object-Reminder Condition

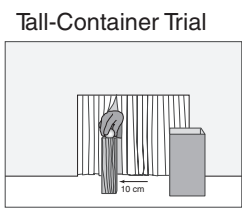

Short-Container Trial

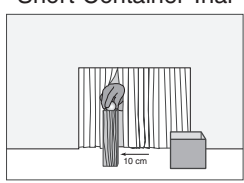

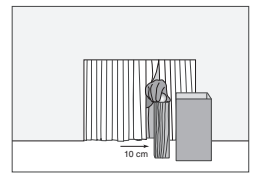

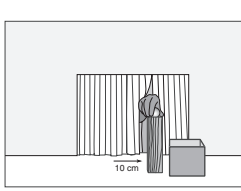

Figure 3. Schematic drawings of the reminder events presented to the infants during the 20-s delay in Experiment 3.

delay. If priming dissipated because of the fading of the object index or height representation, then watching a new set of stimuli in motion during the delay should not help extend the effect of priming beyond the delay. As a result, the infants should still fail to detect the violation. In contrast, if priming dissipated because information was no longer bound to the index, then the occlusion reminder might prompt the infants to do so during the delay, enabling them to detect the violation when they watched the target events.

However, different results across the two reminder conditions, if obtained, could come from different numbers of stimuli involved. One could argue that events involving four items were more engaging to infants than events involving only two items. Or, perhaps Object 2 and the screen served as reference points (e.g., Duffy, Huttenlocher, LeVine, \& Duffy, 2005) that helped infants encode height information of the target object and container. To address these issues, a control condition was conducted (see Figure 4, Number-Control Condition), in which Object 2 and the screen stood to the left of the target object and container and remained stationary throughout the trial. During the delay, infants watched the same movement as in the object-reminder condition with the target object. If the effect of priming could be prolonged simply by adding Object 2 and the screen as reference points or as extra stimuli to engage infants' attention, then infants in this control condition should also succeed in detecting the violation in the target events.

Yet another control condition was conducted (see Figure 4, Occlusion-Control Condition) to directly test whether the reminder effect, if obtained in the occlusion-reminder condition, was derived from the occlusion component. In this control condition, a very short Object 2 was moved back and forth above the screen, creating a reminder that was perceptually similar to the occlusion reminder but involved minimal occlusion. If the reminder effect was derived from the occlusion component, priming should still dissipate after the 20-s delay in the occlusion-control condition, and infants should still fail to detect the violation.

\section{Method}

Participants. Participants were 56 healthy term infants (25 female, 31 male; range $=4$ months 0 days to 5 months 19 days). They were randomly assigned to the occlusion-reminder $(M=4$ months 21 days), object-reminder ( $M=4$ months 20 days), number-control ( $M=4$ months 20 days), or occlusion-control ( $M=4$ months 29 days) conditions. An additional seven infants were tested but their data excluded for inattentiveness or fussiness $(n=5)$, observer error $(n=1)$, or parental interference $(n=1)$.
Occlusion-Control Condition

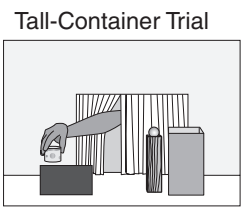

Short-Container Trial

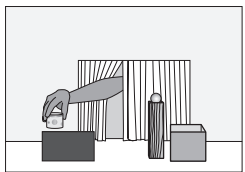

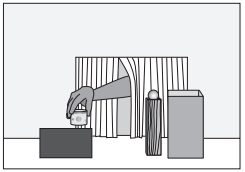

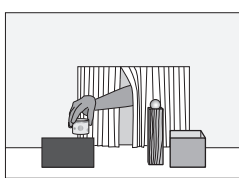

Number-Control Condition

Tall-Container Trial

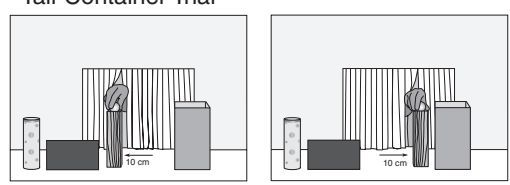

Short-Container Trial
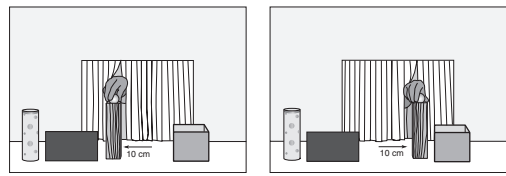

Figure 4. Schematic drawings of the reminder event in the occlusion-control and number-control conditions of Experiment 3. 
As in Experiment 2, half of the infants received a short-container trial, and half received a tall-container trial.

Apparatus, events, and procedure. The following modifications were introduced in Experiment 3. In the occlusionreminder condition, two additional stimuli were present throughout the trial (see Figure 3). A black screen $(16 \mathrm{~cm}$ wide $\times 9 \mathrm{~cm}$ high) stood $17 \mathrm{~cm}$ to the left of the container; a spotted cylinder (Object 2, $15 \mathrm{~cm}$ high and $5 \mathrm{~cm}$ in diameter) rested sideways behind the screen and out of the infant's view. During the 20-s delay, the experimenter first placed Object 2 upright behind the right edge of the screen $(2 \mathrm{~s})$. Next, she slid Object 2 to the left edge of the screen ( $1 \mathrm{~s})$; after a pause ( $1 \mathrm{~s})$, she slid Object 2 to the right edge of the screen $(1 \mathrm{~s})$, followed by another pause $(1 \mathrm{~s})$. The 4-s sliding movement was conducted for a total of four cycles. At the end of the delay, Object 2 was returned to its starting position (2s).

In the object-reminder condition, the experimenter first returned the target object to its starting position ( $1 \mathrm{~s}$ ) and paused (1 s). Next, it was slid $10 \mathrm{~cm}$ to the left $(1 \mathrm{~s})$, followed by another pause $(1 \mathrm{~s})$. In the remainder of the delay, the target object was slid back and forth between the starting and left positions for four cycles.

In the number-control condition, the screen stood $17 \mathrm{~cm}$ to the left of the container, as in the occlusion-reminder condition; however, Object 2 stood $2 \mathrm{~cm}$ to the left of the screen throughout the trial to eliminate the occlusion component. During the delay, the experimenter slid the target object back and forth as in the objectreminder condition.

Finally, in the occlusion-control condition, a new Object 2 was used; it was identical to the original Object 2 except for its height $(4.5 \mathrm{~cm})$. When the new Object 2 was $1.5 \mathrm{~cm}$ above the screen, its top was $6 \mathrm{~cm}$ above the screen, rendering an event perceptually highly similar to the occlusion reminder (compare Figure 3 and Figure 4). At the beginning of the trial, Object 2 was placed behind the screen as in the occlusion-reminder condition. During the delay, the experimenter first placed it $1.5 \mathrm{~cm}$ above the right edge of the screen $(2 \mathrm{~s})$. Next, it was moved horizontally back and forth above the screen (16 s). At the end of the delay, it was returned to the starting location behind the screen $(2 \mathrm{~s})$. Note that when Object 2 was emerging from behind the screen or being lowered to its initial position behind the screen, infants might spot a very brief occlusion configuration between Object 2 and the screen. (A frame-by-frame analysis of the recordings of the event showed that the total duration lasted only about $0.87 \mathrm{~s}$.)

After the 20-s delay, all of the infants watched the target, containment events as in Experiment 2. Interobserver agreement during the target events ranged from $89 \%$ to $100 \%$ and averaged $97 \%$ per infant.

\section{Results and Discussion}

Infants' looking times at the target event were entered into three $2 \times 2$ ANOVAs to compare the occlusion-reminder condition with three other conditions separately. The ANOVA included condition (occlusion-reminder versus each of the other conditions) and trial type (tall- or short-container trial) as between-subjects factors. Each of the analyses yielded a significant Condition $\times$ Trial Type interaction; occlusion-reminder versus object-reminder $F(1,24)=$ $4.91, p<.05$; occlusion-reminder versus number-control $F(1$, $24)=5.49, p<.05$; occlusion-reminder versus occlusion-control
$F(1,24)=6.12, p<.025$. Planned comparisons indicated that in the occlusion-reminder condition, infants who received the shortcontainer trial looked reliably longer $(M=78.47 \mathrm{~s}, S E=1.43 \mathrm{~s})$ than did those who received the tall-container trial $(M=41.90 \mathrm{~s}$, $S E=9.89 \mathrm{~s}$, Cohen's $d=1.96)$. In contrast, infants in the other conditions tended to look equally in the short- and tall-container trials (object-reminder $M \mathrm{~s}=51.67$ and $54.11 \mathrm{~s}, S E \mathrm{~s}=10.61$ and $9.20 \mathrm{~s}$, respectively, $d=0.09$; number-control $M \mathrm{~s}=53.84$ and $53.30 \mathrm{~s}, S E \mathrm{~s}=8.62$ and $7.90 \mathrm{~s}, d=0.02$; occlusion-control $M \mathrm{~s}=$ 43.77 and $46.54 \mathrm{~s}, S E \mathrm{~s}=8.07$ and $9.37 \mathrm{~s}, d=0.12$; all $F \mathrm{~s}>8.62$, $p$ s <.05). Nonparametric Wilcoxon rank-sum tests confirmed these results (occlusion-reminder $W=33.5, p<.025$; objectreminder $W=51, p>.10$; number-control $W=51.5, p>.10$; occlusion-control $W=52, p>.10)$.

The results in Experiment 3 suggested that watching additional occlusion events during the 20-s delay, even if they were conducted with stimuli different from the target object and container, primed the infants to detect the height violation in the containment event. In contrast, and quite surprisingly, continued exposure to the target object and container during the delay with the absence of occlusion did not facilitate infants' use of information about these stimuli, as shown in the object-reminder condition. These results suggested that the occlusion prime might enhance infants' detection of physical violations not by strengthening the memory of relevant information but by increasing the likelihood of retrieving the information and binding it to the object index. These findings were particularly striking because introducing two additional objects (Object 2 and the screen) would have increased the task demand, yet enhancement was still observed in the occlusionreminder condition.

The infants in the two control conditions failed to detect the violation, suggesting that (a) adding Object 2 and the screen alone or (b) watching a perceptually highly similar reminder that involved a minimal amount of occlusion was not enough to extend priming beyond a 20 -s delay. Thus, the positive results obtained in the occlusion-reminder condition were not derived from the increased number of stimuli involved, the presence of reference points, or the superficial perceptual aspects of the reminder event. Together, the results of Experiment 3 suggested that watching occlusion events, even with a new set of stimuli, strengthened the effect of priming, whereas watching the target or a different object being moved in a nonocclusion manner did not.

The present findings implied that priming effects can be "spilled over" to bystander objects that are not directly involved in an event. Such spill-over effects have obvious value in helping infants direct their attention broadly to relevant information about the objects that are currently used and those that may be potentially used in the future. The more general priming effects turn out to be, the more likely such processes may represent the kinds of experience infants naturally encounter and rely on for developing their ability to select and use task-relevant information.

The spill-over effects demonstrated here suggest the need to modify the three-system account, in particular, on how the objecttracking system interacts with the object-representation and physical-reasoning systems. The present findings suggest that priming could occur not necessarily through binding height information about a particular object to a particular object index. Instead, infants are primed to use task-relevant information through strengthening a general tendency to retrieve the informa- 
tion from the object-representation system and bind it to the object index for the purpose of physical reasoning such that information about a wider array of objects could be used for a longer period of time. The extent to which priming effects can be spilled over to bystander objects or even to the objects that are initially absent is an open question of theoretical importance. Based on the threesystem model, priming effects should only be carried over to objects that are initially present and hence have been indexed in the object-tracking system.

\section{General Discussion}

The present research showed that although 4.5-month-old infants do not spontaneously detect a violation of height in containment events, exposure to occlusion events for $24 \mathrm{~s}$ seemed enough to facilitate their detection of such a violation (Experiment 1), and the effect appeared to last at least $8 \mathrm{~s}$ but dissipated by $20 \mathrm{~s}$ (Experiment 2). In addition, the results suggested that another occlusion hint, even if it involved new stimuli, facilitated infants' use of information about the original set of stimuli, whereas continued exposure to the original stimuli in a nonocclusion context did not (Experiment 3). Together with previous research (Wang \& Baillargeon, 2005), priming seems relatively general, applying across a variety of events and ages, and appears to last over seconds. In addition, the enhancement seems to arise from increased likelihood of retrieval, rather than strengthened memory of objects.

Broadly, the present research showcases the powerful influence of experience on the development of infant cognition and, more specifically, on how infants come to use task-relevant information in their observation of unfamiliar physical events. In the following sections, I discuss the limitations and implications of the present findings, linking the research to three important areas of infant cognition: information processing, memory reactivation, and learning mechanisms.

\section{Considering an Alternative Model}

The three-system model (e.g., Baillargeon et al., 2009; Wang \& Baillargeon, 2008b) provides a theoretical framework that motivated the priming experiments presented here. However, an alternative, information-processing model is worth consideration. According to this alternative model, watching occlusion events may highlight certain dimensions of an object (e.g., height information) for infants, thus increasing the likelihood for them to use the information.

The present research provided compelling evidence that it was the occlusion component of the priming event that drove the enhancement effect. For example, infants in the occlusion-control condition of Experiment 3 watched a nonocclusion reminder that was perceptually highly similar to the occlusion reminder and still failed to use height information. Thus, it is clear that without considering what infants bring to bear when watching occlusion events, a general information-processing model cannot explain why occlusion was the necessary component for the enhancement effect. In contrast, the three-system model and, in particular, the physical reasoning system provides a clearer explanation for why infants' use of object information can be facilitated by watching some but not other types of events. Specifically, it clarifies the way in which infants distinguish different types of physical events and the process by which infants learn to use object information for a particular kind of event category, and evidence has been obtained to support this account. First, the categorization literature showed that infants distinguish between different spatial relations and form abstract categories of physical events (e.g., Casasola, 2005). Second, prior training studies demonstrated that deliberate exposure to examples of one event category can teach infants to use object information when they encounter another example of the same category, but learning is not generalized to a different event category (e.g., Wang \& Baillargeon, 2008a; Wang \& Kohne, 2007). Together, these findings support that when processing physical events, infants rely on a top-down process that brings to bear their existing knowledge about the events.

Nonetheless, it is important to point out that further evidence is needed to determine whether the object-tracking system is indeed necessary and whether it can be replaced by an informationhighlighting account. The present findings on its own cannot rule out the alternative explanation that occlusion (but not nonocclusion) events may increase the salience of height information and therefore promote infants' use of the information in subsequent events. However, recent evidence on negative priming appears to challenge the information-highlighting account. For example, Li, Baillargeon, and Simons (cited in Baillargeon et al., 2009) showed that infants who normally were capable of using height information in occlusion events ceased to do so if they first watched covering events in which they normally neglected to use height information. Based on the information-highlighting account, occlusion events should have made height information salient whether or not they were preceded by covering events. On the other hand, these findings are consistent with the three-system model. Specifically, watching covering events does not prompt infants to retrieve and include height information in the representation of the first, covering event. When infants use the same object index to process the second, occlusion event, no information about object height could be carried forward to the representation of the occlusion event; as a result, infants now failed to use the information that they otherwise would have considered.

\section{Priming Through Reactivating a Retrieval Tendency}

Existing literature on infants' use of object information suggests that even though infants are capable of maintaining object representations over a short period of time, whether they use these representations is affected by various contextual factors (e.g., Baillargeon et al., 2009; Carey \& Xu, 2001; Wang \& Mitroff, 2009; Xu \& Carey, 1996). The present research complements this literature and suggests that neighboring event contexts may affect infants' use of information. Experience watching events from a category for which infants have learned to use certain object information can induce in infants the tendency to retrieve the information and use it in subsequent events. Moreover, multiple exposures to events from the category for which the information has already been identified as relevant can serve as a hint to increase the likelihood of retrieving the information and elevate the effect of priming.

The results of Experiment 3 suggest that priming and target events need not involve the same stimuli and that infants appear able to retrieve variable information for stimuli in use and for 
stimuli not in use but present in the event. The present finding seems contradictory to that of Wang and Baillargeon (2005), in which watching an occlusion event with a blue or yellow object failed to prime 8-month-old infants to use height information in a covering event that involved a red object. However, the infants in Wang and Baillargeon (2005) watched only one set of priming events prior to target events. In the present research, the infants watched two sets of priming events: The first set involved the target object and container, and the second set involved Object 2 and the screen. After watching the first set of priming events, height information has been bound to the indexes of the target stimuli. It could be that reactivated retrieval and binding can be applied to a wider range of stimuli present in the event, including the stimuli currently not in use. It follows that such a spillover effect should not occur with a single set of priming events. To test this possibility, future research can modify Experiment 3 by replacing the first set of occlusion priming events with events for which infants have not yet identified height as a relevant variable. For example, 4.5-month-olds could watch (a) covering events (the variable height is typically not identified until infants are about 12 months) involving target stimuli, (b) occlusion events involving Object 2 and the screen, and finally (c) containment events involving target stimuli. Evidence that infants fail to use height information in containment events despite the presence of occlusion priming events with Object 2 will support the above speculation.

Future research can increase the number of stimuli to investigate the limitation of the spillover effect-the phenomenon that priming with Object 1 helps infants use information about Object 2. On the basis of the literature of performance signatures in infancy (e.g., Feigenson, Dehaene, \& Spelke, 2004), it seems unlikely for infants to retrieve variable information and bind it to all of the object indexes when the number exceeds four. Under this condition, further research can examine whether infants will select a smaller set of object indexes to retrieve information for and what may affect their selective retrieval.

\section{Priming Versus Other Enhancement Effects}

The priming effect shown in the present research appears similar to memory reactivation (e.g., Rovee-Collier \& Cuevas, 2009) on the surface, but it has some distinctive features. In memory reactivation, exposure to the environmental context in which a task was initially learned serves as a reminder and primes infants to regain their access to a forgotten memory (e.g., Borovsky \& RoveeCollier, 1990; Rovee-Collier, Griesler, \& Earley, 1985; RoveeCollier, Sullivan, Enright, Lucas, \& Fagen, 1980), and increased exposure to the prime increases the reactivation (e.g., Bearce \& Rovee-Collier, 2006; Hayne, Gross, Hildreth, \& Rovee-Collier, 2000). The priming phenomenon is similar to memory reactivation in that exposure to the learning context facilitates retrieval in a different context, and multiple exposures to the prime increase performance at test. However, the cognitive process underlying priming may be different from memory reactivation. So far, priming has been investigated on infants' ability to select a particular piece of information and use it to detect violations of physical rules or principles, whereas memory reactivation examines infants' memory about their own behavior and the outcome associated with the behavior (e.g., their kicking causes a crib mobile to move). To link the priming research to the issue of memory reactivation more directly, a new experiment is currently underway that investigates whether similar priming can occur in a memory task. Rather than detecting a violation, infants are asked to remember an object feature and determine whether the feature has changed after a delay. Preliminary data so far suggest that after watching occlusion events, 4.5-month-olds become better at detecting a change to the object's height in the context of containment events (Wang \& Onishi, 2010).

Compared with the priming phenomenon shown here, a more robust enhancement on infants' use of task-relevant information has been demonstrated (Wang \& Baillargeon, 2008a). Ninemonth-olds, who have not learned that height is relevant for predicting covering events, watched multiple exemplars of covering events. Such experience "taught" them to identify the variable height, and the infants became able to use height information when they encountered another covering event. The effect of teaching lasted at least $24 \mathrm{hr}$. In addition, facilitation occurred even when training and testing differed in many aspects (e.g., different rooms, objects, nature of the task; Wang \& Kohne, 2007). The contrast between the robustness of priming and teaching suggests that different mechanisms may be involved. Whereas priming may enable infants to transfer information and biases (e.g., the bias to retrieve height information) from one event to another, it does not allow infants to learn about the relevance of a new variable for the target event. Future work can vary environmental contexts (e.g., using different apparatuses) across priming and testing phases to find out whether priming still occurs when the context changes. Investigations of this sort will shed important light onto learning mechanisms in infancy.

\section{Conclusions}

Although further evidence is still needed to rule out the information-highlighting account, the present research makes it clear that priming can occur not by strengthening a memory trace but by improving the retrieval of relevant object features. The findings presented here underscore the remarkable flexibility of infants' representational process. Even young infants appear to attend to detailed aspects of the spatial context in which an object is moved. Their use of object information in an event can be affected by prior experience with another event.

\section{References}

Acredolo, L. P., Adams, A., \& Goodwyn, S. W. (1984). The role of self-produced movement and visual tracking in infant spatial orientation. Journal of Experimental Child Psychology, 38, 312-327. doi:10.1016/ 0022-0965(84)90128-0

Aguiar, A., \& Baillargeon, R. (2002). Developments in young infants' reasoning about occluded objects. Cognitive Psychology, 45, 267-336. doi:10.1016/S0010-0285(02)00005-1

Amso, D., \& Johnson, S. P. (2006). Learning by selection: Visual search and object perception in young infants. Developmental Psychology, 42, 1236-1245.

Bahrick, L. E. (2002). Generalization of learning in three-and-a-halfmonth-old infants on the basis of amodal relations. Child Development, 73, 667-681. doi:10.1111/1467-8624.00431

Baillargeon, R., \& DeVos, J. (1991). Object permanence in young infants: Further evidence. Child Development, 62, 1227-1246. doi:10.2307/1130803

Baillargeon, R., Li, J., Ng, W., \& Yuan, S. (2009). An account of infants' physical reasoning. In A. Needham \& A. Woodward (Eds.), Learning 
and the infant mind (pp. 66-116). New York, NY: Oxford University Press.

Baillargeon, R., \& Wang, S. (2002). Event categorization in infancy. Trends in Cognitive Sciences, 6, 85-93. doi:10.1016/S13646613(00)01836-2

Barrett, T. M., Davis, E. F., \& Needham, A. (2007). Learning about tools in infancy. Developmental Psychology, 43, 352-368. doi:10.1037/00121649.43.2.352

Bearce, K. H., \& Rovee-Collier, C. (2006). Repeated priming increases memory accessibility in infants. Journal of Experimental Child Psychology, 93, 357-376. doi:10.1016/j.jecp.2005.10.002

Borovsky, D., \& Rovee-Collier, C. (1990). Contextual constraints on memory retrieval at 6 months. Child Development, 61, 1569-1583. doi: $10.2307 / 1130765$

Bushnell, E. W., \& Boudreau, J. P. (1993). Motor development and the mind: The potential role of motor abilities as a determinant of aspects of perceptual development. Child Development, 64, 1005-1021. doi: $10.2307 / 1131323$

Campos, J. J., Anderson, D. I., Barbu-Roth, M. A., Hubbard, E. M., Hertenstein, M. J., \& Witherington, D. (2000). Travel broadens the mind. Infancy, 1, 149-219. doi:10.1207/S15327078IN0102_1

Carey, S., \& Xu, F. (2001). Infants' knowledge of objects: Beyond object files and object tracking. Cognition, 80, 179-213.

Casasola, M. (2005). When less is more: How infants learn to form an abstract categorical representation of support. Child Development, 76, 279-290. doi:10.1111/j.1467-8624.2005.00844.x

Dueker, G. L., Modi, A., \& Needham, A. (2003). 4.5-month-old infants' learning, retention and use of object boundary information. Infant Behavior \& Development, 26, 588-605. doi:10.1016/j.infbeh.2003.05.002

Duffy, S., Huttenlocher, J., LeVine, S., \& Duffy, R. (2005). How infants encode spatial extent. Infancy, 8, 81-90. doi:10.1207/ s15327078in0801_5

Feigenson, L., Dehaene, S., \& Spelke, E. (2004). Core systems of number. Trends in Cognitive Sciences, 8, 307-314. doi:10.1016/j.tics.2004 .05 .002

Hauf, P., Elsner, B., \& Aschersleben, G. (2004). The role of action effects in infant's action control. Psychological Research, 68, 115-125. doi: 10.1007/s00426-003-0149-2

Hayne, H., Barr, R., \& Herbert, J. (2003). The effect of prior practice on memory reactivation and generalization. Child Development, 74, 16151627. doi: $10.1046 / j .1467-8624.2003 .00627 . x$

Hayne, H., Gross, J., Hildreth, K., \& Rovee-Collier, C. (2000). Repeated reminders increase the speed of memory retrieval by 3-month-old infants. Developmental Science, 3, 312-318. doi:10.1111/1467-7687.00125

Hespos, S. J., \& Baillargeon, R. (2001). Infants' knowledge about occlusion and containment events: A surprising discrepancy. Psychological Science, 12, 141-147. doi:10.1111/1467-9280.00324

Hespos, S. J., \& Baillargeon, R. (2006). Décalage in infants' knowledge about occlusion and containment events: Converging evidence from action tasks. Cognition, 99, B31-B41. doi:10.1016/j.cognition.2005.01.010

Huettel, S. A., \& Needham, A. (2000). Effects of balance relations between objects on infants' object segregation. Developmental Science, 3, 415427. doi:10.1111/1467-7687.00136

Kellman, P. J., \& Arterberry, M. E. (1998). The cradle of knowledge: Development of perception in infancy. Cambridge, MA: MIT Press.

Learmonth, A. E., Lamberth, R., \& Rovee-Collier, C. (2004). Generalization of deferred imitation during the first year of life. Journal of Experimental Child Psychology, 88, 297-318. doi:10.1016/ j.jecp.2004.04.004

Leslie, A. M., Xu, F., Tremoulet, P. D., \& Scholl, B. J. (1998). Indexing and the object concept: Developing "what" and "where" system. Trends in Cognitive Sciences, 2, 10-18. doi:10.1016/S1364-6613(97)01113-3

Luo, Y., \& Baillargeon, R. (2007). Do 12.5-month-old infants consider what objects others can see when interpreting their actions? Cognition, 105, 489-512.

Needham, A. (2000). Improvements in object exploration skills may facilitate the development of object segregation in early infancy. Journal of Cognition and Development, 1, 131-156. doi:10.1207/ S15327647JCD010201

Needham, A., Barrett, T., \& Peterman, K. (2002). A pick me up for infants' exploratory skills: Early simulated experiences reaching for objects using "sticky" mittens enhances young infants' object exploration skills. Infant Behavior \& Development, 25, 279-295. doi:10.1016/S01636383(02)00097-8

Noles, N. S., Scholl, B. J., \& Mitroff, S. R. (2005). The persistence of object file representations. Perception \& Psychophysics, 67, 324-334.

Pylyshyn, Z. W. (2001). Visual indexes, preconceptual objects, and situated vision. Cognition, 80, 127-158. doi:10.1016/S0010-0277(00) 00156-6

Rigney, J., \& Wang, S. (2009, April). Behind versus inside: Infants form an abstract category of containment events. Poster presented at the biennial meeting of the Society for Research in Child Development, Denver, CO.

Rovee-Collier, C., \& Cuevas, K. (2009). Multiple memory systems are unnecessary to account for infant memory development: An ecological model. Developmental Psychology, 45, 160-174. doi:10.1037/a0014538

Rovee-Collier, C., Griesler, P. C., \& Earley, L. A. (1985). Contextual determinants of retrieval in three-month-old infants. Learning and Motivation, 16, 139-157. doi:10.1016/0023-9690(85)90009-8

Rovee-Collier, C., Sullivan, M. W., Enright, M. K., Lucas, D., \& Fagen, J. W. (1980). Reactivation of infant memory. Science, 208, 1159-1161. doi:10.1126/science.7375924

Scholl, B. J., \& Leslie, A. M. (1999). Explaining the infants' object concept: Beyond the perception/cognition dichotomy. In E. Lepore \& Z. Pylyshyn (Eds.), What is cognitive science? (pp. 26-73). Oxford, England: Blackwell.

Sommerville, J. A., Hildebrand, E. A., \& Crane, C. C. (2008). Experience matters: The impact of doing versus watching on infants' subsequent perception of tool-use events. Developmental Psychology, 44, 12491256. doi: $10.1037 / \mathrm{a} 0012296$

Sommerville, J. A., Woodward, A. L., \& Needham, A. (2005). Action experience alters 3-month-old infants' perception of others' actions. Cognition, 96, B1-B11. doi:10.1016/j.cognition.2004.07.004

Soska, K. C., Adolph, K. E., \& Johnson, S. P. (2010). Systems in development: Motor skill acquisition facilitates 3D object completion. Developmental Psychology, 46, 129-138. doi:10.1037/a0014618

Wang, S. H., \& Baillargeon, R. (2005). Inducing infants to detect a physical violation in a single trial. Psychological Science, 16, 542-549. doi:10.1111/j.0956-7976.2005.01572.x

Wang, S. H., \& Baillargeon, R. (2006). Infants' physical knowledge affects their change detection. Developmental Science, 9, 173-181. doi: 10.1111/j.1467-7687.2006.00477.x

Wang, S. H., \& Baillargeon, R. (2008a). Can infants be "taught" to attend to a new physical variable in an event category? The case of height in covering events. Cognitive Psychology, 56, 284-326. doi:10.1016/ j.cogpsych.2007.06.003

Wang, S. H., \& Baillargeon, R. (2008b). Detecting impossible changes in infancy: A three-system account. Trends in Cognitive Sciences, 12, 17-23. doi:10.1016/j.tics.2007.10.012

Wang, S. H., \& Kohne, L. (2007). Visual experience enhances 9-month-old infants' use of task-relevant information in an action task. Developmental Psychology, 43, 1513-1522. doi:10.1037/0012-1649.43.6.1513

Wang, S. H., \& Mitroff, S. R. (2009). Preserved visual representations despite change blindness in infants. Developmental Science, 12, 681687. doi:10.1111/j.1467-7687.2008.00800.x

Wang, S. H., \& Onishi, K. H. (2010). Priming 4-month-old infants to detect changes in physical events. Manuscript in preparation.

Wilcox, T., \& Chapa, C. (2004). Priming infants to attend to color and 
pattern information in an individuation task. Cognition, 90, 265-302. doi:10.1016/S0010-0277(03)00147-1

Wilcox, T., Woods, R., \& Chapa, C. (2008). Color-function categories that prime infants to use color information in an object individuation task. Cognitive Psychology, 57, 220-261. doi:10.1016/j.cogpsych.2008.02.001

Wilcox, T., Woods, R., Chapa, C., \& McCurry, S. (2007). Multisensory exploration and object individuation in infancy. Developmental Psychology, 43, 479-495. doi:10.1037/0012-1649.43.2.479
Xu, F., \& Carey, S. (1996). Infants' metaphysics: The case of numerical identity. Cognitive Psychology, 30, 111-153. doi:10.1006/cogp .1996 .0005

Received March 13, 2009

Revision received May 3, 2010

Accepted June 21, 2010 